\title{
Dynamics of Arachidonic Acid Metabolism in Macrophages From Delayed-Type Hypersensitivity (Schistosoma mansoni egg) and Foreign-Body-Type Granulomas
}

\author{
Stephen W. Chensue, Denise A. Ellul, Mary Spengler, Gene I. Higashi, \\ and Steven L. Kunkel \\ Departments of Pathology and Epidemiology, University of Michigan Medical \\ School (S.W.C., D.A.E., M.S., S.L.K.), and School of Public Health, University of \\ Michigan (G.I.H.), Ann Arbor
}

The present study examines the kinetics of arachidonic acid (AA) metabolism by murine macrophages isolated from sites of experimentally induced pulmonary granulomatous inflammation. Macrophages of T-cell-mediated hypersensitivity lesions induced by Schistosoma mansoni eggs (SE-GM) and non-T-cellmediated foreign-body-type lesions (FB-GM) induced by Sephadex beads were examined. Overall, macrophages from both types of lesions produced mainly lipoxygenase pathway metabolites, leukotrienes, and monohydroxyeicosatetraenoic acids (mono-HETEs). Early after induction (4 days [4D]), SE-GM showed an augmented zymosan-stimulated AA release and metabolism compared to resident peritoneal macrophages. Macrophages from mature lesions (8-32D) showed constitutive synthesis of metabolites and were refractory to zymosan stimulation. Both SE-GM and FB-GM showed augmented AA uptake incorporating a large proportion into neutral lipids. A direct comparison of SEGM and FB-GM revealed that the T-cell-mediated lesion produced lesser amounts of prostaglandins and leukotrienes and showed reduced incorporation of AA into phosphatidylcholine. These data suggest that AA metabolism by granuloma macrophages is sequentially modified during recruitment and activation at sites of chronic inflammation.

Key words: macrophages, arachidonic acid, granuloma, leukotrienes, monohydroxyeicosatetraenoic acids, Schistosoma

\section{INTRODUCTION}

Numerous in vitro studies have established the exquisite capacity of macrophages to metabolize arachidonic acid (AA) to a variety of biologically active com-

Received April 3, 1985; accepted June 18, 1985.

Reprint requests: S.W. Chensue, Department of Pathology, University of Michigan Medical School, Box 045, Medical Science I, 1315 Catherine Road, Ann Arbor, MI 48109.

(C) 1985 Alan R. Liss, Inc. 
pounds [1-4]. These include products derived from lipoxygenase (LTs, HETEs) and cyclooxygenase (PGs) enzyme systems. Previous studies of Scott et al [5] and Humes et al [6] showed that mouse macrophage metabolic capacity is dependent on their state of activation such that there is diminished AA metabolism in activated cells. The significance of these findings with regard to macrophage inflammatory function is not known. Moreover, few studies have defined the sequential changes in macrophage AA metabolism occurring at sites of chronic inflammation.

In the present study, we examined AA metabolism by murine macrophages isolated from synchronously developing puylmonary granulomas induced by Schistosoma mansoni eggs (SE-GM) or Sephadex beads (FB-GM). The former represents a T-cell-mediated hypersensitivity-type lesion under regulation of $T$ helper and suppressor cell subsets $[7,8]$. The latter is a foreign-body-type lesion without antigen-specific T-cell involvement. Thus, we determined sequential changes in AA metabolism of macrophages at sites of chronic inflammation recruited under contrasting conditions.

Our results showed time-dependent changes in macrophage AA metabolism as granulomas matured and resolved. Overall, the macrophages produced predominantly lipoxygenase-derived metabolites. Zymosan-stimulated macrophages from early lesions (4 days) showed an augmented release of the products compared to resident peritoneal macrophages. Those from mature lesions (8-32D) synthesized these products constitutively and were unresponsive to phagocytic stimuli. Granuloma macrophages also showed greater incorporation of AA with a large proportion found in neutral lipids. When provided with exogenous AA, macrophages from non-T-cell lesions produced greater amounts of LTs and PGs compared to those of T-cellmediated lesions, which produced primarily HETEs. Also, macrophages from T-cell lesions showed less incorporation of fatty acid (AA and oleic acid) into phosphatidylcholine. These findings suggest that AA metabolism by granuloma macrophages is sequentially modified during macrophage recruitment and activation at sites of granulomatous inflammation.

\section{MATERIALS AND METHODS}

\section{Animals}

Pathogen-free female CBA/J mice (The Jackson Laboratory, Bar Harbor, ME) were used throughout this study. The mice were maintained under standard care and given food and water ad libitum.

\section{Induction of Pulmonary Granulomas}

Eggs were isolated aseptically from the livers of mice previously infected with 200 cercariae of Schistosoma mansoni by the method of Coker and von Lichtenberg [9]. The isolated schistosome eggs were suspended to $6,000 \mathrm{eggs} / \mathrm{ml}$ sterile physiological saline and each mouse received 3,000 eggs via tail vein injection. Sephadex beads (G-200 superfine, Pharmacia, Uppsala, Sweden) were swollen in saline and sized with nylon mesh to remove particles less than $40 \mu \mathrm{m}$ in diameter. The slurry was autoclaved and then suspended to 10,000 beads $/ \mathrm{ml}$ of sterile saline. Each mouse received 5,000 beads by tail vein injection as above.

\section{Isolation and Culture of Granuloma Macrophages}

Dispersed granuloma cells were obtained from schistosome egg or Sephadex bead-induced lung granulomas by a modification of the procedure of Moore et al 
[10]. At 4, 8, 16, or 32 days postembolization mice were sacrificed and granulomatous lungs were inflated with $1 \mathrm{ml}$ cold RPMI-1640, excised, and homogenized for $20 \mathrm{sec}$ at low speed in a commercial Waring blender. Granulomas were then collected and rinsed free of adherent blood, single cells, and parenchyma over a stainless steel mesh. Granulomas were washed three times in RPMI, pelleted in a 50-ml plastic centrifuge tube (Corning Glass Works, Corning, NY), and resuspended in three volumes of sterile digestion medium consisting of RPMI containing $10 \%$ fetal bovine serum (FBS) (KC Biological, Inc., Lenexa, KS), $10 \mathrm{mM}$ HEPES buffer, 2,000 U/ml Type II collagenase (Sigma Chemical Co., St. Louis, MO), and $20 \mu \mathrm{g} / \mathrm{ml}$ gentamicin (Schering Pharmaceutical Corp., Kenilworth, NJ). The dispersed cells were then vigorously agitated for $30 \mathrm{~min}$ in a $37^{\circ} \mathrm{C}$ water bath. The suspension was passed through a \#100 steel mesh and collected by centrifugation. The resuspended cells were strained through a 50- $\mu \mathrm{m}$ nylon sieve to remove nondispersed cells and particulates. Granuloma cells were washed twice, centrifuged, counted, and resuspended in RPMI containing $10 \%$ FBS and $20 \mu \mathrm{g} / \mathrm{ml}$ gentamicin. Two milliliters of granuloma cells $\left(2 \times 10^{6}\right.$ cells $\left./ \mathrm{ml}\right)$ were allowed to adhere to $60-\mathrm{mm}$ plastic culture dishes (Corning Glass Works) overnight at $37^{\circ} \mathrm{C}$ in a $5 \% \mathrm{CO}_{2}$, humidified atmosphere. Nonadherent cells were removed by at least two vigorous rinses with warm serumfree medium. The number of adherent cells was quantitated by direct counting of Wright-stained monolayers. These cells are routinely $>90 \%$ macrophages by morphological criteria, phagocytosis of inert beads, and staining for nonspecific esterase [11].

In some experiments resident peritoneal macrophages were used as control cells and were obtained by the standard method of peritoneal lavage with sterile RPMI medium. Collagenase treatment of suspended peritoneal macrophages had no effect on their capacity to metabolize arachidonic acid.

\section{Determination of Pulmonary Granuloma Area}

Mice were sacrificed at $4,8,16$, and 32 days postegg challenge and the lungs were inflated with $10 \%$ buffered formalin, excised, and prepared for histologic section. The pulmonary granuloma areas were measured from hematoxylin and eosin stained sections using an Omicron Alpha Image Analyzer (Bausch and Lomb, Rochester, NY) interfaced with an attached computer. A minimum of 20 granulomas were measured from each lung. Granulomas were measured from at least five mice per data point.

\section{Determination of Cyclooxygenase/Lipoxygenase-Derived Arachidonic Acid Metabolites}

The washed macrophage monolayers $(60-\mathrm{mm}$ dishes) were overlaid with $2.0 \mathrm{ml}$ of serum-free RPMI containing $1 \mu \mathrm{Ci}$ of ${ }^{3}[\mathrm{H}]$ arachidonic acid $\left({ }^{3}[\mathrm{H}] \mathrm{AA}\right)$ (New England Nuclear, Boston, MA, specific activity $=91 \mathrm{Ci} / \mathrm{mmol}$ ). After a 4-hr incubation at $37^{\circ} \mathrm{C}$, the media were discarded and monolayers were washed twice with prewarmed RPMI to remove unincorporated ${ }^{3}[\mathrm{H}] \mathrm{AA}$. Two milliliters of fresh RPMI was added and the cells were incubated for another $45 \mathrm{~min}$ with or without opsonized zymosan $(500 \mu \mathrm{g})$. In some experiments monolayers were simply overlaid with ${ }^{3}[\mathrm{H}] \mathrm{AA}$ medium and accumulated metabolites were measured at 6,12 , and $18 \mathrm{hr}$.

Arachidonic acid metabolites were extracted as previously described [12]. Media and monolayers were collected after incubation, acidified to pH 3.0 with $1 \mathrm{~N}$ 
$\mathrm{HCl}$, then extracted twice with three volumes of ethyl acetate. This procedure gave greater than $90 \%$ recovery of all AA metabolite standards. The solvent was vaporized under vacuum using a Speed Vac Concentrator (Savant Instruments, Inc., Westbury, $\mathrm{NY}$ ). Extracts were stored under $100 \% \mathrm{~N}_{2}$ at $-20^{\circ} \mathrm{C}$ and then dissolved in $0.1 \mathrm{ml}$ absolute methanol just before column chromatography. Metabolites of ${ }^{3}[\mathrm{H}] \mathrm{AA}$ were identified by reverse-phase high-performance liquid chromatography (HPLC) utilizing two solvent systems. In method I, 0.1-ml samples were applied to a 5- $\mu \mathrm{m} \mathrm{C-18}$ $(30 \times .4-\mathrm{cm})$ column (Varian, Palo Alto, CA) at $29^{\circ} \mathrm{C}$ with a $1-\mathrm{ml} / \mathrm{min}$ flow rate. The solvent, acetonitrile (Burdick Jackson, Muskegon, MI)/water/trifluoroacetic acid (pH 2.0) (Pierce Chemical Co., Rockford, IL), was used as follows: the first $\mathbf{4 0}$ min were isocratic $(31: 69: 0.1, \mathrm{vol} / \mathrm{vol} / \mathrm{vol})$ followed by a linear gradient increase of acetonitrile starting at 45:55:0.1 to 65:35:0.1 over a 2-hr period. Finally the column was washed for $30 \mathrm{~min}$ at 100:0:0.1. In method II, a 3- $\mu \mathrm{m} \mathrm{C-18} \mathrm{column} \mathrm{(Varian)} \mathrm{was}$ used under similar conditions but the solvents were run as follows: isocratic for 35 min (33:67:0.1), then a linear gradient from 45:55:0.1 to 55:45:01 over $1 \mathrm{hr}$, then a second 10-min gradient from 55:45:01 to 80:20:01, and finally 100:0:0.1 to remove AA and wash the column. The eluates were continuously monitored for UV absorbance using a variable wavelength detector $(280$ and $235 \mathrm{~nm})$. In addition, radioactivity was measured in an in-line radioactive monitor (Radiomatic Instruments \& Chemical Co. Inc., Westshore, FL) and a flow ratio of 5:1 (aqueous scintillant [Radiomatic, Tampa, FL] to sample) was used. Characteristic ultraviolet spectra of leukotrienes and mono-HETEs were determined with a Carey 210 spectrophotometer and compared with known standards.

The columns were calibrated using radiolabeled and unlabeled standards. Thromboxane $B_{2}$, prostaglandins $E_{2}, F_{2 \alpha}$, and $D_{2}$, as well as 5- and 12-HETE were kindly provided by Drs. John Pike and Robert Gorman (The Upjohn Co., Kalamazoo, MI). Unlabeled leukotrienes were the generous gifts of Dr. J. Rokach (Merck-Frosst Laboratories, Quebec, Canada). Carbon-14-labeled LTB $_{4}$ was provided by Henry Showell (Pfizer, Central Research, Groton, CT). Tritiated LTC $4,{ }^{14}[\mathrm{C}]$ oleic acid, and ${ }^{3}$ [H]AA were purchased from New England Nuclear. Fifteen-HPETE and 15-HETE were prepared using soybean lipoxygenase (Sigma) as described [13]. In some cases unlabeled standards were added to samples to act as internal markers.

\section{Determination of AA Incorporation Into Membrane Phospholipids}

At designated intervals following exposure to ${ }^{3}[\mathrm{H}] \mathrm{AA}$, granuloma macrophage monolayers were washed twice with warm RPMI and then placed on ice. The cells were then scraped from the dishes using a rubber policeman and quantitated. Cells were pelleted by centrifugation and dissolved in chloroform $(4 \mathrm{ml})$-methanol $(1 \mathrm{ml})$. After a $15-\mathrm{min}$ incubation at room temperature, $1.25 \mathrm{ml}$ of $5 \% \mathrm{KCl}$ solution was added and the mixture was vortexed for $30 \mathrm{sec}$, then centrifuged $(500 \mathrm{~g}, 5 \mathrm{~min})$. The organic layer was collected and vaporized under $\mathrm{N}_{2}$. The residue was then analyzed for lipids by silica gel thin-layer chromatography (TLC) (EM reagents, Darmstadt, Germany) with a two solvent system as follows: 1) chloroform-methanol acetic acid ( $100 \mathrm{ml} \mathrm{CHCl} 3,60 \mathrm{ml} \mathrm{CH} 3 \mathrm{OH}, 16 \mathrm{ml}$ glacial acetic acid, $8 \mathrm{ml} \mathrm{H}_{2} \mathrm{O}$ ), followed by 2) petroleum ether-ethyl ether (78:22). Pure lipid standards served as markers (Supelco, Inc., Belefonte, PA). Potassium iodide was used to stain and localize the separated lipids. 


\section{Statistical Analysis}

The Student's t-test was used to analyze control and experimental groups where applicable. Values of $p>.05$ were considered not significant.

\section{RESULTS}

\section{Spontaneous Metabolism of AA by Granuloma Macrophage Populations}

Initially, we wished to determine the profile of metabolites spontaneously released by granuloma macrophages. In these experiments macrophage cultures were prelabeled with $1.0 \mu \mathrm{Ci}$ of ${ }^{3}[\mathrm{H}] \mathrm{AA}$ for $4 \mathrm{hr}$, then washed and allowed to incubate a further $45 \mathrm{~min}$. Following incubation, cultures were extracted and analyzed by HPLC as described in Materials and Methods. As shown in Figure 1, time-dependent changes were observed in SE-GM. At day 4 the major products were free AA and a single mono-HETE that comigrated with the 12-HETE standard. At days 8 and 16 a second mono-HETE as well as material in the leukotriene (LT) region could be

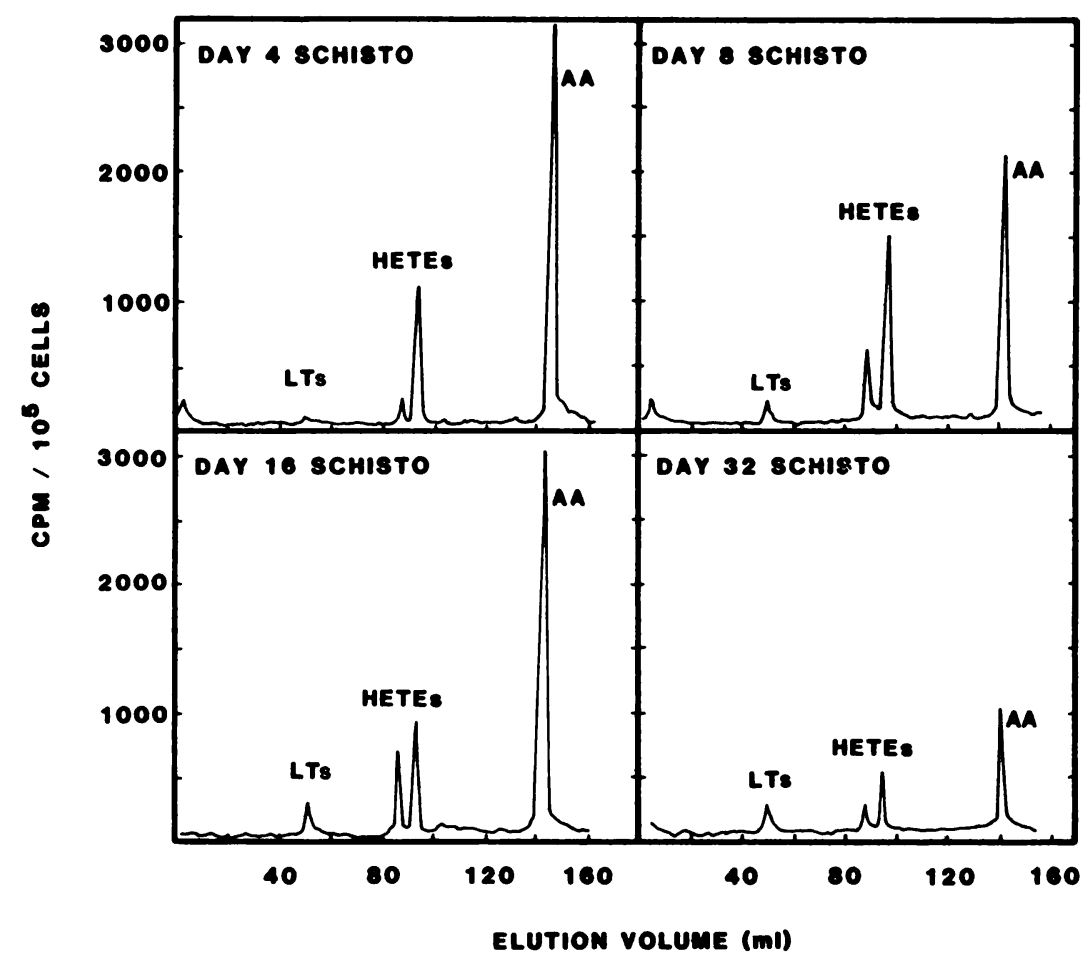

Fig. 1. Spontaneous release of arachidonic acid metabolites by macrophages from S. mansoni egg (hypersensitivity-type) granulomas at sequential stages of development. Macrophage cultures were prelabeled $(4 \mathrm{hr})$ with $1 \mu \mathrm{Ci}^{3}[\mathrm{H}]$ arachidonic acid, washed, incubated an additional $45 \mathrm{~min}$, then extracted for HPLC analysis (method I). Total cpm of the material was normalized to $1 \times 10^{5}$ cells and replotted. There was no statistical difference in AA uptake by macrophages at the indicated time points. Chromatograms represent the mean of three separate experiments. 


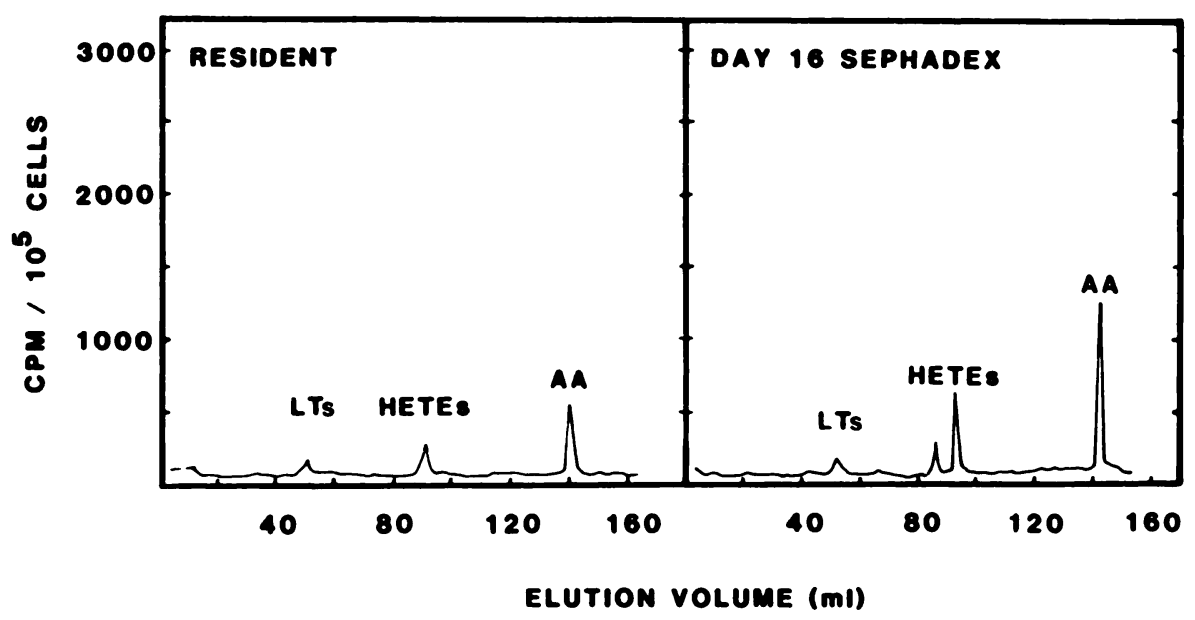

Fig. 2. Spontaneous release of arachidonic acid metabolites by resident peritoneal and 16D Sephadex bead (foreign-body-type) granuloma macrophages. Cultures and extractions were prepared as described for Figure 1. Uptake of AA by resident macrophages was approximately $50 \%$ of that for bead granuloma macrophages.

detected. By day 32, when the lesion was resolving, there was a notable reduction in HETEs and free AA but a persistence of material in the LT region.

We likewise examined the products released spontaneously by resident peritoneal and FB-GM macrophages (Fig. 2). As previously reported, unstimulated resident macrophage produced barely detectable amounts of material $[5,12]$. However, 16D FB-GM gave a pattern similar to that obtained with the 32D SE-GM.

\section{Effect of Exogenous Stimuli on Granuloma Macrophage AA Release and Metabolism}

We next determined the products released by granuloma macrophages following stimulus with opsonized zymosan. Figure 3 shows the zymosan-induced AA release response of SE-GM compared to that of resident peritoneal macrophages. On a percent basis, there was a steady decline in AA release as the granuloma reached maximal size (day 16) (compare upper and central panels). However, examination of the total counts per minute (CPM) of AA released showed this effect was due to an increase in the spontaneous release of AA (lower panel). At day 4 there was actually a dramatic augmentation of the total amount of released AA, which declined significantly by day 8 and reached a minimum by day 32 .

Analysis of the AA metabolites produced by SE-GM in response to zymosan gave results similar to that observed with AA release (Fig. 4). The spontaneous synthesis of LTs increased to a maximum at day 16 and was unchanged thereafter. Mono-HETE production reached a maximum at day 8 and declined as the lesion resolved (day 32). In contrast, the zymosan-induced release of these products was greatest at day 4 with little or no significant incremental synthesis thereafter.

Thus, SE-GM early in development showed an augmented AA release and metabolism in response to zymosan compared to resident peritoneal macrophages, 


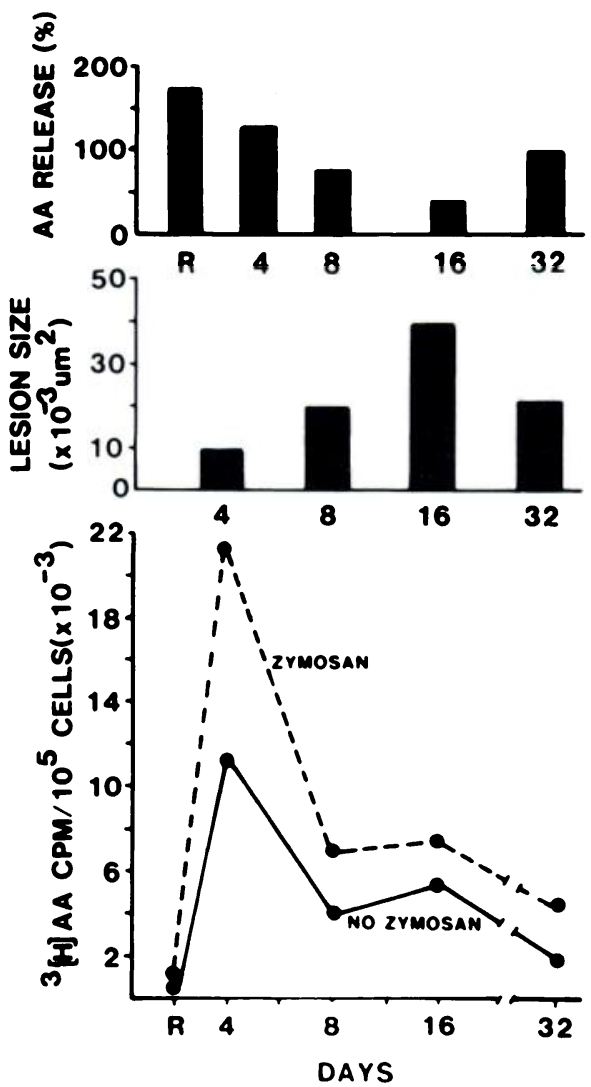

Fig. 3. Zymosan-induced arachidonic acid release from schistosome egg granuloma macrophages during sequential stages of lesion development. Percent AA release (upper panel) of resident and ichistosome egg granuloma macrophages. Granuloma size (middle panel) determined from groups of five mice. Total cpm of AA recovered by HPLC from unstimulated ( _ (O---) cultures (lower panel). Cultures were labeled with $1 \mu \mathrm{Ci}$ of ${ }^{3}[\mathrm{H}] \mathrm{AA}$ for $4 \mathrm{hr}$, washed, then stimulated $45 \mathrm{~min}$ with opsonized zymosan. Normal mouse resident (R) peritoneal macrophages served as a control. AA release values were derived from 3 to 4 separate experiments.

while macrophages of more mature lesions showed a constitutive release of significant amounts of AA metabolites, but they had blunted responses to a phagocytic stimulus.

An examination of FB-GM also revealed blunted responses to zymosan. Figure 5 compares the HPLC profiles of the AA metabolites produced by resident peritoneal and 16D FB-GM in the presence or absence of zymosan. The spontaneous release of AA and the major metabolite, mono-HETE, was 10-30-fold greater for macrophages from bead lesions. Upon stimulation with zymosan, resident macrophages showed a $200 \%$ increase in AA release with synthesis of two major metabolites, $\mathrm{PGE}_{2}$ and LTC $_{4}$, as reported by others [14]. In contrast, the FB-GM showed only a $40 \%$ increase in AA release with little increase in other metabolites.

The above results suggested that macrophages at mature (8-32D) hypersensitivity or foreign-body-type granulomas begin constitutive release of AA and its metabolites, but they show little or no incremental synthesis with further stimulation. 


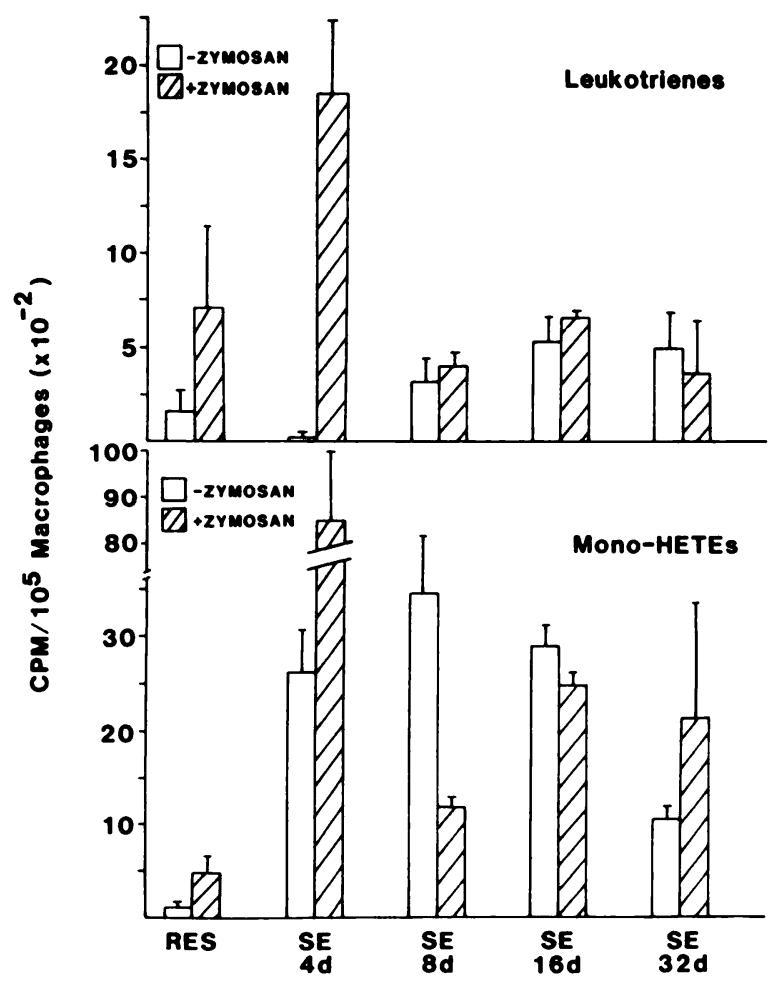

Fig. 4. Zymosan-induced release of leukotrienes and mono-HETEs from schistosome egg granuloma macrophages during sequential stages of development. Macrophage cultures were labeled with $1 \mu \mathrm{Ci}$ of ${ }^{3}[\mathrm{H}] \mathrm{AA}$ for $4 \mathrm{hr}$, washed, then incubated for $\mathbf{4 5} \mathrm{min}$ with or without zymosan. Extracted cultures were analyzed by HPLC (method I) and the total cpm of recovered products were determined and normalized to cell number. Bars show the mean $\pm \mathrm{SE}$ of 3 to 4 separate experiments. Values of resident peritoneal cultures (RES) are shown for comparison.

\section{Metabolism of Exogenous AA by Granuloma Macrophages}

We next compared the capacity of SE-GM and FB-GM to metabolize exogenously provided $\mathrm{AA}$. In these experiments macrophage monolayers were overlaid with serum-free media containing $1.0 \mu \mathrm{Ci}^{3}[\mathrm{H}] \mathrm{AA}$; then at designated intervals the cultures were extracted and analyzed for the presence of metabolites. Figure 6 shows the results of these experiments. The exogenously supplied AA was converted to essentially the same major products seen in the shorter 45-min cultures but in greater quantities. Over an 18-hr period, 16D FB-GM synthesized greater amounts of PG and LT from exogenously supplied AA compared to 16D SE-GM. The total quantity of mono-HETEs produced was comparable among the groups, but there was a tendency for macrophages from egg lesions to make more mono-HETEs. These differences were enhanced by providing larger amounts $(5 \mu \mathrm{Ci})$ of ${ }^{3}[\mathrm{H}] \mathrm{AA}$ as shown in the HPLC chromatograms (Fig. 7). As before, macrophages from Sephadex bead lesions produced more PGs and LTs and significantly less mono-HETEs than those of hypersensitivity egg lesions.

By adding unlabeled AA ( $5 \mu \mathrm{M})$, enough material was obtained for spectrophotometric analysis of the products (Fig. 8). Two peaks (the major one at 54-59 min 


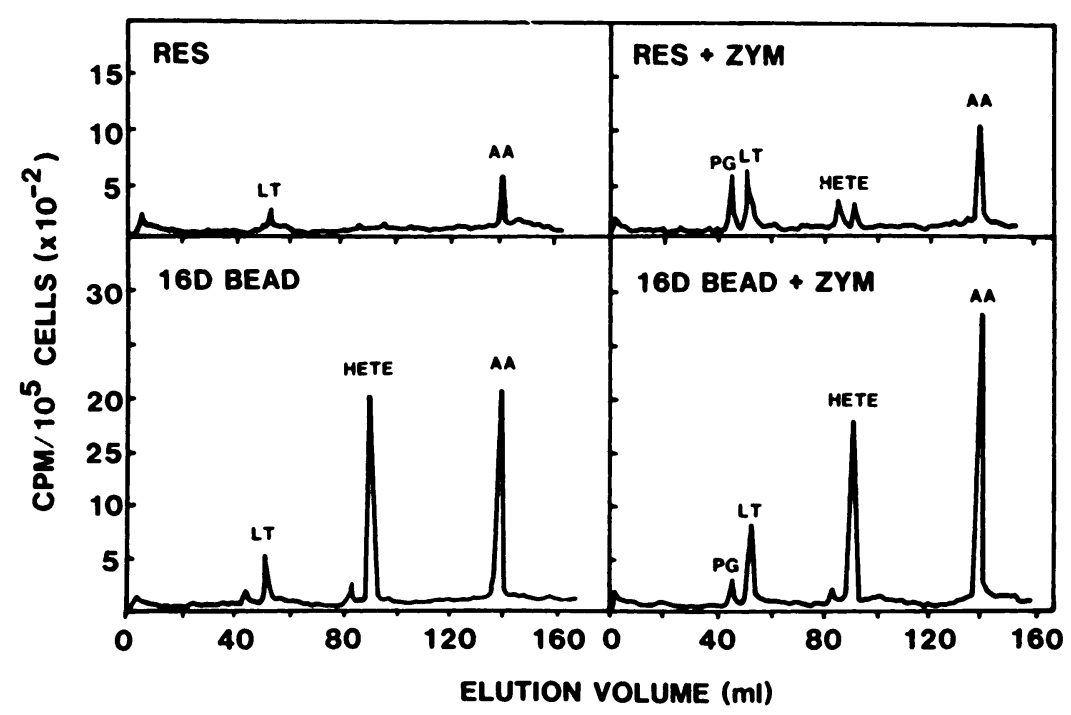

Fig. 5. Zymosan-induced release of arachidonic acid metabolites by resident peritoneal and Sephadex bead (foreign-body-type) granuloma macrophages. Cultures were prelabeled for $4 \mathrm{hr}$ with $1 \mu \mathrm{Ci}$ of ${ }^{3}$ [H]AA, washed, then incubated for $\mathbf{4 5} \mathrm{min}$ with or without zymosan. Extracted cultures were analyzed by HPLC (method I). Chromatograms were derived from two separate experiments.

and a smaller one at 60-65 min) showed characteristic absorbance spectra for SRS-A and $\mathrm{LTB}_{4}$ conjugated trienes, with peak absorbances at 279 and $270 \mathrm{~nm}$, respectively. Likewise, the putative mono-HETE peaks (80-95 min) showed characteristic peak absorbance at $235 \mathrm{~nm}$.

\section{Uptake and Phospholipid Distribution of AA in Granuloma Macrophages}

In view of the changes observed in AA metabolism by granuloma macrophages, it was important to establish the intracellular fate of exogenously supplied AA. Figure 9 compares the uptake of AA by granuloma and resident peritoneal macrophages. A nearly twofold greater amount of AA was incorporated by both the SE- and FB-GM. This is in accord with previous reports demonstrating enhanced AA incorporation by recruited macrophage populations [2].

Using thin-layer chromatography, the distribution of ${ }^{3}[\mathrm{H}] \mathrm{AA}$ among membrane lipids was determined (Fig. 10). Among granuloma macrophages, up to $60 \%$ of the incorporated label was found in neutral lipids (NL), 25-30\% in phosphatidylcholine (PC), and the remainder in phosphatidylinositol (PI), phosphatidylserine (PS), and phosphatidylethanolamine (PE). In contrast, in resident peritoneal macrophages, greater than $80 \%$ of the label was in PC and other phospholipids with only $15 \%$ in NL. Thus, while granuloma macrophages incorporated greater quantities of AA, the bulk of this was placed into neutral lipid pools. Essentially, identical results were obtained when cells were labeled in the presence of albumin (data not shown).

Figure 10 also shows that compared to the other populations, SE-GM had consistently less AA present in PC $(p<.01)$. Moreover, we could demonstrate loss of AA from PC, PI, and PS when cells were stimulated with zymosan. An approxi- 


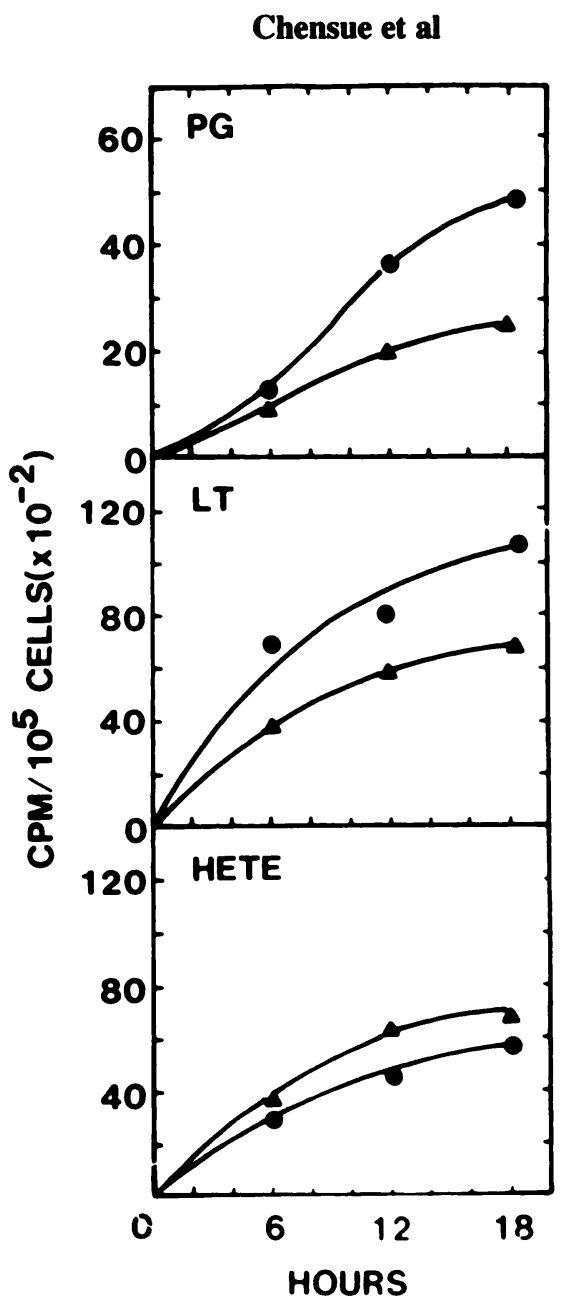

Fig. 6. Kinetics of arachidonic acid metabolism by macrophages from 16D schistosome egg and Sephadex bead granulomas. Macrophage cultures were overlaid with medium containing $1 \mu \mathrm{Ci}$ of ${ }^{3}[\mathrm{H}] \mathrm{AA}$ and then extracted at 6,12 , and $18 \mathrm{hr}$ of incubation. Accumulation products were determined by HPLC (method I) and then normalized to cell numbers. Values were derived from two separate experiments. $\Delta$, schistosome egg; $\odot$, Sephadex bead.

mately $50 \%$ loss was shown for residents, but there was only a $20 \%$ loss for $16 \mathrm{D}$ granuloma macrophages (no figure). When we likewise examined the distribution of another fatty acid, ${ }^{14} \mathrm{C}$-labeled oleic acid, both resident and SE-GM showed comparable uptake into NL (Fig. 11). However, the SE-GM again showed reduced incorporation into PC. Taken together, these data indicated that resident macrophages preferentially placed AA into phospholipids. In contrast, SE-GM showed reduced incorporation into PC with increased incorporation into NL. By the intensity of lipid staining (Fig. 11), granuloma macrophages had increased amounts of triglycerides as previously reported for other "recruited" macrophage populations [2]. Thus, the increased incorporation into NL may in part be due to the increased triglyceride pools. 


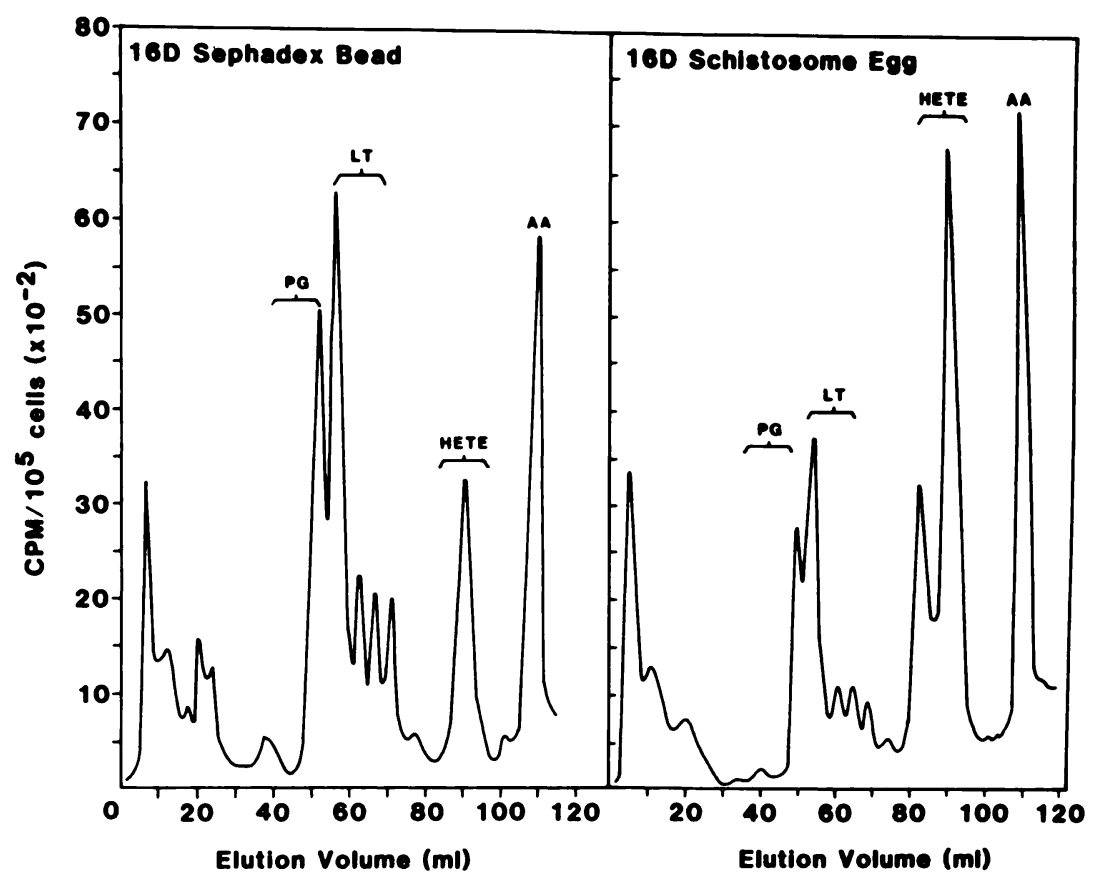

Fig. 7. Exogenous metabolism of arachidonic acid by Sephadex bead (FB-GM) and schistosome egg (SE-GM) granuloma macrophages. Macrophage cultures were overlaid with $5 \mu \mathrm{Ci}$ of ${ }^{3}[\mathrm{H}] \mathrm{AA}$ and then extracted after $12 \mathrm{hr}$ of culture. Representative HPLC chromatograms (method II) are shown with the total integrated cpm per peak.

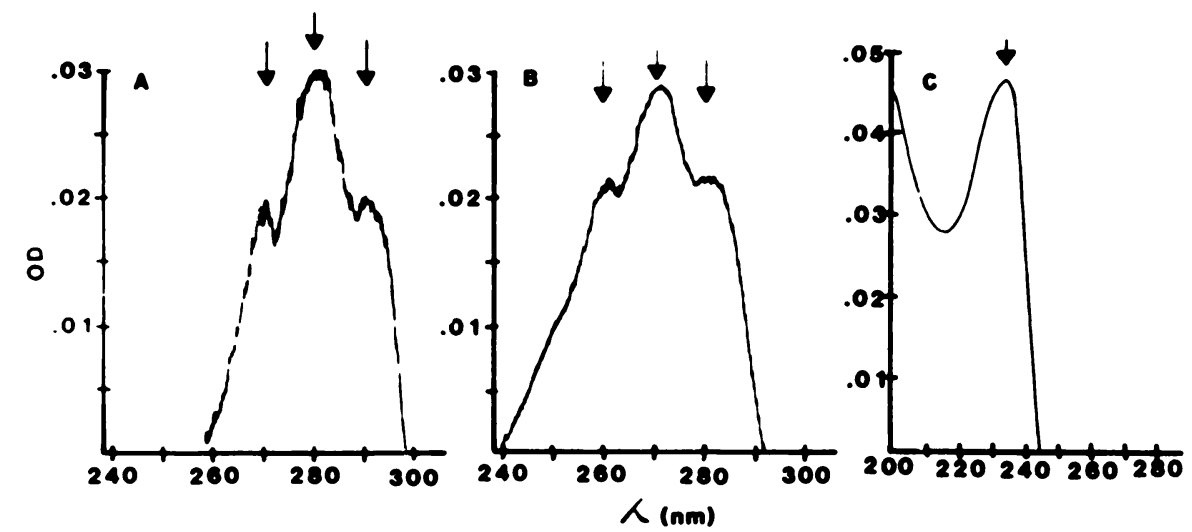

Fig. 8. Spectrophotometric analysis of granuloma macrophage arachidonic acid metabolites. Eight-day schistosome egg granuloma macrophage cultures were overlaid with medium containing $5 \mu \mathrm{M}$ unlabeled AA and incubated for $12 \mathrm{hr}$. Following extraction, the metabolites were analyzed by HPLC (method II) and the peaks were collected for spectrophotometric scanning. Material eluted from 54 to $59 \mathrm{~min}$ (A), 60 to $65 \mathrm{~min}(\mathrm{~B})$, and 80 to $95 \mathrm{~min}(\mathrm{C})$. 


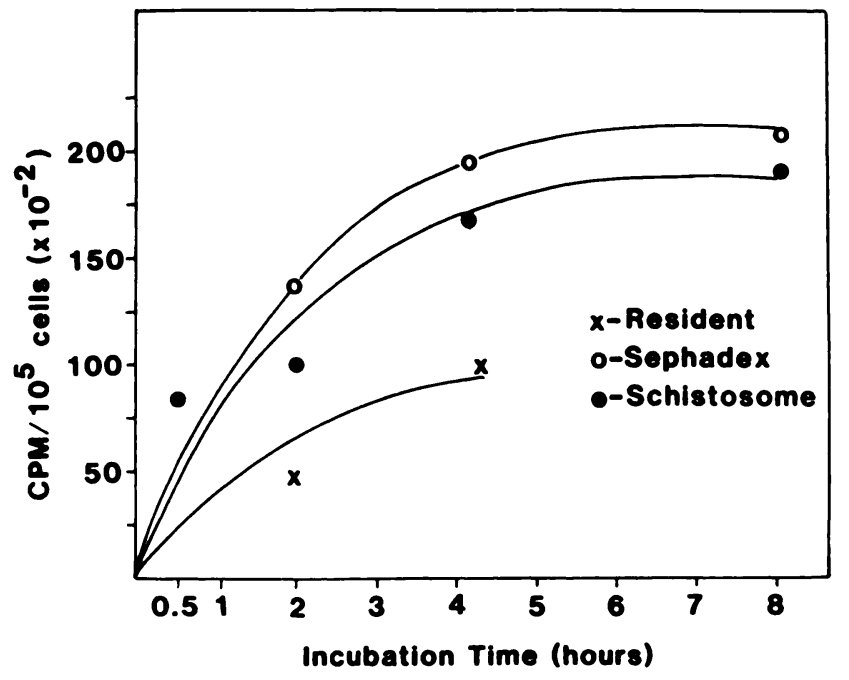

Fig. 9. Uptake of arachidonic acid by resident peritoneal and granuloma (16D) macrophage populations. Macrophage cultures were overlaid with $1 \mu \mathrm{CI}{ }^{3}[\mathrm{H}] \mathrm{AA}$. At designated intervals monolayers were washed, collected, solubilized in chloroform-methanol, and aliquots were measured for total radioactivity. Points represent means of three experiments.

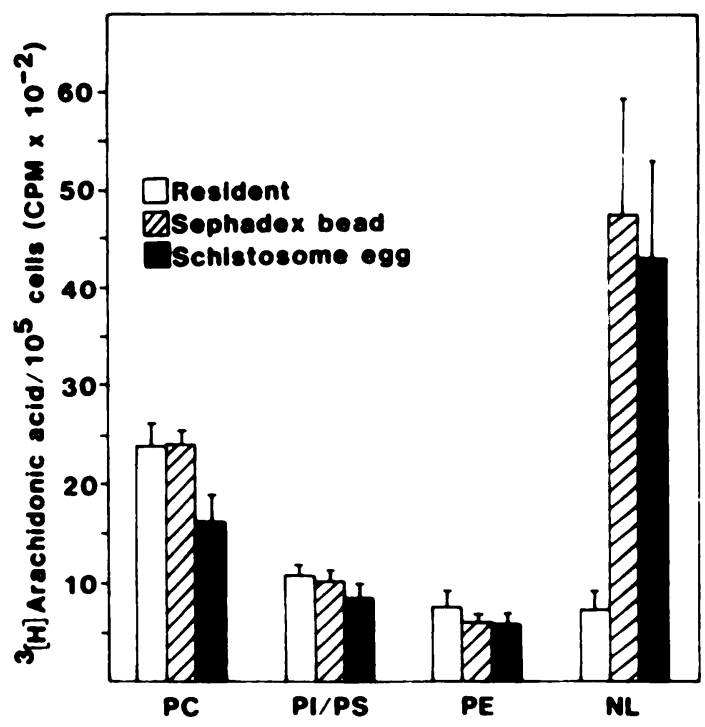

Fig. 10. Distribution of arachidonic acid in membrane lipids of resident peritoneal and granuloma (16D) macrophage populations. Macrophage cultures were labeled with $1 \mu \mathrm{Ci}^{3}[\mathrm{H}] \mathrm{AA}$ for 120 min and membrane lipids were then analyzed by TLC as described in Materials and Methods. Total radioactivity was measured in iodine stained bands scraped from the plates. Bars represent the mean $\pm S E$ of three experiments. 


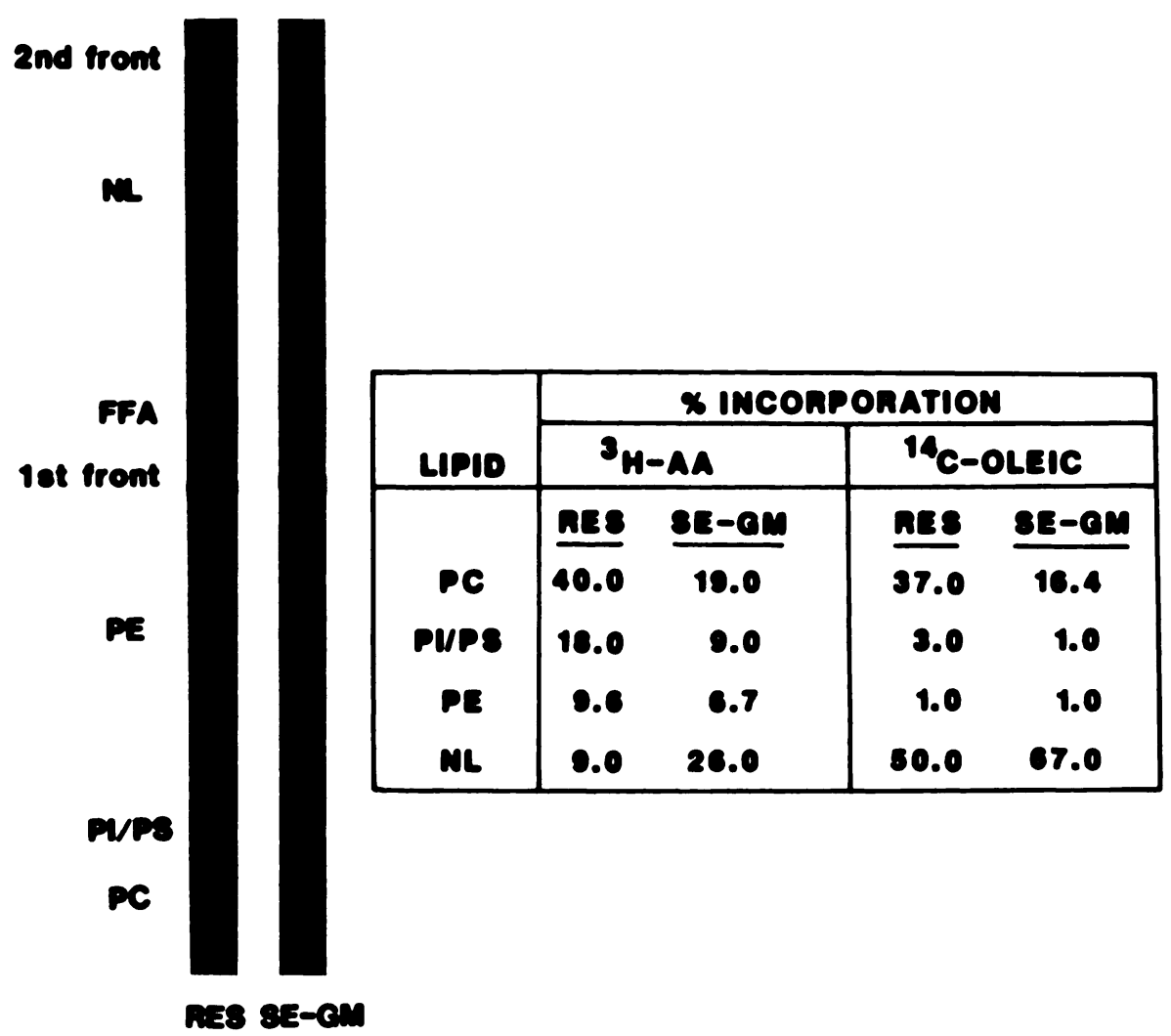

Fig. 11. Distribution of oleic and arachidonic acid in membrane lipids of resident peritoneal and schistosome egg granuloma (16D) macrophages. Characteristic lipid chromatograms of resident peritoneal and SE-GM are shown; the material was extracted from $360 \times 10^{3}$ and $140 \times 10^{3}$ cells, respectively. Despite less applied material, the SE-GM show more intense staining of neutral lipids. The percent distribution of ${ }^{14}[\mathrm{C}]$ oleic acid and ${ }^{3}[\mathrm{H}] \mathrm{AA}$ in major lipids is shown in the table.

\section{DISCUSSION}

The potential importance of AA metabolites in the induction and regulation of inflammation has become increasingly apparent [15-18]. Thus, many investigators have attempted to define their production and function at sites of inflammation in order to aid the therapeutic manipulation of destructive inflammatory processes.

In the present study employing a model of synchronous murine pulmonary granulomatous inflammation, we have defined the AA metabolites produced by macrophages (thought to be important sources of these products and the major component of granulomas) during the induction, maintenance, and resolution of these chronic lesions. Our results show that granuloma macrophages (GM) actively metabolize AA into a variety of products but primarily those derived from lipoxygenases, LTs, and HETEs. This is in accord with our previous study [12], which examined hepatic granulomas during active murine schistosomiasis mansoni, as well as studies of Humes et al and Bonta et al $[19,20]$ with carrageenin-induced granulomas.

When compared to stimulated resident peritoneal macrophages, GM did not produce significant amounts of PGs after 45 min of culture. This finding agrees with 
previous reports of Humes et al [6] and Scott et al [5], which showed reduced PG production by recruited or "activated" macrophages. It further supports the notion that substrate availability or the activity of cyclooxygenase (CO) pathway enzymes is "down-regulated" in activated macrophages. This also seems consistent with our finding that FB-GM showed greater PG synthesis than hypersensitivity SE-GM, which are thought to be more "activated" $[11,21,22]$.

It is conceivable that down-regulation of PG synthesis may be an important proinflammatory event. While PGs, especially of the E series, may have vasoactive functions in early acute inflammatory events, they appear to suppress many inflammatory responses such as lymphocyte proliferation [15], lymphokine production [23], granulocyte function [24], macrophage Ia antigen expression [21,25], and, as we recently reported, interleukin-1 production [26]. Thus, specific reduction in PG synthesis by macrophages may help inflammatory events to proceed.

The predominance of lipoxygenase (LO)-derived AA metabolite synthesis by GM is consistent with accumulating evidence showing their proinflammatory function [27-29]. We reported that pharmacologic suppression of LO but not CO products caused a significant reduction of granulomatous inflammation [30]. Likewise, others have shown that carrageenin-induced granulomas were not affected by $\mathrm{CO}$ inhibitors [31]. Hence, LO products may be important to the induction and maintenance of the cellular infiltrate. One caveat should be added. Until the precise functions of the LO products, especially the HETEs, have been better characterized, it will be difficult to fully understand the significance of their presence at inflammatory sites.

Our finding showing augmented zymosan-induced release of AA and its metabolites by GM from early lesions followed by refractory responses in mature lesions may indicate that macrophage AA metabolism is tailored to the specific phase of inflammation. Teleologically, it seems appropriate that during the early recruitment phase macrophages would be "primed" for maximal mediator release in response to stimuli. Subsequently, these cells shift to constitutive production of these mediators for maintenance of the lesion. This refractory state of "activated" macrophages has been described in other systems and may reflect prior in vivo triggering followed by metabolic modulation [5]. This modulation may be related to the release of macrophage-derived reactive oxygen intermediates $\left(\mathrm{eg} \mathrm{O}_{2}^{-}\right)$, which we have recently shown to become significant at day 8 and beyond [32]. It has been suggested that such products may inactivate enzymes involved in AA metabolism [33]. This could explain the refractory state as well as the observed suppression of AA metabolism with zymosan stimulation at day 8 . Heretofore, this refractory state has seemed paradoxical since "activated" cells were expected to show augmented responses to stimuli. Our data suggest that this may in fact be true if macrophages are examined soon after recruitment to inflammatory foci. Moreover, special attention must be paid to quantitating the spontaneous release and absolute amounts of products rather than only percentage shifts.

Analysis of AA uptake and its distribution revealed notable differences between resident and granuloma macrophage populations. As reported for other "activated" macrophage populations [2], both the SE-GM and FB-GM showed enhanced incorporation of AA. Much of the additional incorporated AA was found in NL (triglycerides/cholesterol esters). Examination of phospholipids showed that the total CPM incorporated was comparable for resident and FB-GM, but there was significantly reduced incorporation into PC by SE-GM. The latter finding and the above-mentioned 
reduction in PG and LT synthesis were the main characteristics of SE-GM distinguishing them from FB-GM. Presumably, this difference is related to the presence of antigen-specific T-cell factors present at SE but not FB granulomas. It is noteworthy that at the resolution phase (32D), SE-GM AA metabolism appeared to revert to a pattern resembling the FB-GM. Thus, as the antigenic stimulus of $\mathrm{T}$ cells "burns out," the lesion may become FB-like.

It is tempting to draw a relationship between the reduced LT/PG synthesis and the decreased amounts of AA in phosphatidylcholine. At least two scenarios can be constructed. 1) There is evidence that AA may be stored in different subcellular pools that are linked to different enzyme systems $[34,35]$. Phosphatidylcholine may be the primary reservoir of AA for LT and PG synthesis, whereas HETE may be derived from additional AA pools, eg, other phospholipids and neutral lipids. Reduced acylation of PC in "activated" SE-GM would thereby lead to reduced LT and PG synthesis. 2) An alternative model is that our data reflect brisk phospholipase $A_{2}$ activity and reduced PG and LT synthesis in the face of active HETE synthesis. Further studies are underway to distinguish these possibilities and to determine the inter- and intracellular signals that modulate granuloma macrophage AA metabolism.

\section{ACKNOWLEDGMENTS}

The authors wish to express their appreciation for the expert secretarial assistance of Jeny Brown. This work was supported by National Institutes of Health grants HL 31237 and HL 30963.

\section{REFERENCES}

1. Humes, J.L., Bonney, R.J., Pelus, L., Dahlgren, Kuehl, F.A., and Davies, P. Macrophages synthesize and release prostaglandins in response to inflammatory stimuli. Nature 269, 149, 1977.

2. Davies, P., Bonney, R.J., Humes, J.L., and Kuehl, F.A. The synthesis of arachidonic acid oxygenation products by various mononuclear phagocyte populations. In Mononuclear Phagocytes (van Furth, R., ed.) The Hague: Nijhoff, p. 1317, 1980.

3. Scott, W.A., Zrike, J.M., Hamill, A.L., Kempe, J., and Cohn, Z.A. Regulation of arachidonic acid metabolites in macrophages. J. Exp. Med. 152, 324, 1980.

4. Chensue, S.W., and Kunkel, S.L. Arachidonic acid metabolism and macrophage activation. Clin. Lab. Med. 3, 677, 1983.

5. Scott, W.A, Pawlowski, N.A., Murray, H.W., Adreach, M., Zrike, J., and Cohn, Z.A. Regulation of arachidonic acid metabolism by macrophage activation. J. Exp. Med. 155, 1148,1982

6. Humes, J.L., Burger, S., Galavage, M., Kuehl, F.A., Jr., Wightman, P.D., Dahlgren, M.E., Davies, P., and Bonney, R.J. The di- minished production of arachidonic acid oxygenated products by elicited mouse peritoneal macrophages: Possible mechanisms. J. Immunol. 124, 2110, 1980.

7. Chensue, S.W., Wellhausen, S.R., and Boros, D.L. Participation of Lyt $1+$ and Lyt 2+ $T$ lymphocytes in the suppression of granuloma formation and lymphokine production in Schistosoma mansoni-infected mice. J. Immunol. 127, 363, 1980.

8. Chensue, S.W., Boros, D.L., and David, C.S. Regulation of granulomatous inflammation in murine schistosomiasis. II. T suppressor cellderived, I-C subregion-encoded soluble suppressor factor mediates regulation of lymphokine production. J. Exp. Med. 157, 219, 1980.

9. Coker, C.M., and von Lichtenberg, F. Revised method for isolation of Schistosoma mansoni eggs for biologic experimentation. Proc. Soc. Exp. Biol. Med. 92, 359, 1956.

10. Moore, D.L., Grove, D.I., and Warren, K.S. The Schistosoma mansoni egg granuloma: Quantitation of cell populations. J. Pathol. 121, 41, 1977.

11. Amsden, A.F., and Boros, D.L. Fc-receptor bearing macrophages isolated from hypersen- 
sitivity-type and foreign body granulomas. Delineation of macrophage dynamics, Fc receptor density/avidity and specificity. Am. J. Pathol. 96, 47, 1979.

12. Chensue, S.W., Kunkel, S.L., Higashi, G.I., Ward, P.A., and Boros, D.L. Production of superoxide anion, prostaglandins, and hydroxyeicosatetraenoic acids by macrophages from hypersensitivity-type (Schistosoma mansoni egg) and foreign body-type granulomas. Infect. Immun. 42, 1116, 1983.

13. Hamberg, M., and Samuelsson, B. On the specificity of the oxygenation of unsaturated fatty acids catalyzed by soybean lipoxyidase. J. Biol. Chem. 242, 5329, 1967.

14. Rouzer, C.A., Scott, W.A., Cohn, Z.A., Blackburn, P., and Manning, J.M. Mouse peritoneal macrophages release leukotriene $C$ in response to a phagocytic stimulus. Proc. Natl. Acad. Sci. U.S.A. 77, 1928, 1980.

15. Goodwin, J.S., and Webb, D.R. Regulation of the immune response by prostaglandins. Clin. Immunol. Immunopathol. 15, 106, 1980.

16. Ford-Hutchinson, A.W., Bray, M.A., Doig, M.V., Shipley, M.E., and Smith, M.J.N. Leukotriene B, a potent chemokinetic and aggregating substance released from plymorphonuclear leukocytes. Nature 286, 264, 1980.

17. Klickstein, L.B., Shapleigh, C., and Goetzl, E.J. Lipoxygenation of arachidonic acid as a source of polymorphonuclear leukocyte chemotactic factors in synovial fluid and tissue in rheumatoid arthritis and spondyloarthritis. J. Clin. Invest. 66, 1166, 1980.

18. Kunkel, S.L., and Chensue, S.W. Prostaglandins and the regulation of the immune response. Adv. Inflamm. Res. 7, 93, 1983.

19. Bonta, I.L., Parnham, M.J., and Bragt, P.C. Arachidonate metabolites and the chronic phase of granulomatous inflammation: In Prostaglandin Synthetase Inhibitors: New Clinical Applications (Ramwell, P., ed.) New York: Alan R. Liss, Inc., p. 129, 1980.

20. Bragt, P.C., and Bonta, I.L. In vivo metabolism of $\left[1-{ }^{14} \mathrm{C}\right]$ arachidonic acid during different phases of granuloma development in the rat. Biochem. Pharmacol. 28, 1581, 1979.

21. Kunkel, S.L., Chensue, S.W., Plewa, M., and Higashi, G.I. Macrophage function in the Schistosoma mansoni egg-induced pulmonary granuloma. Role of arachidonic acid metabolites in macrophage Ia antigen expression. Am. J. Pathol. 114, 240, 1984.

22. Sunday, M.E., Stadecker, M.J., Wright, J.A., Aoki, I., and Dorf, M.E. Induction of immune responses by schistosome granuloma macrophages. J. Immunol. 130, 2413, 1983.
23. Gordon, D., Bray, M.A., and Morley, J. Control of lymphokine production by prostaglandins. Nature 262, 401, 1976.

24. Zurier, R.B., Weissmann, G., Hoffstein, S., Kammerman, S., and Hsin, H.T. Mechanisms of lysosomal release from human leukocytes: II. Effect of cAMP and cGMP, autonomic agonists and agents which affect microtubule function. J. Clin. Invest. 53, 297, 1974.

25. Snyder, P.S., Beller, D.I., and Unanue, E.R. Prostaglandins modulate macrophage Ia expression. Nature 299, 163, 1982.

26. Kunkel, S.L., and Chensue, S.W. Arachidonic acid metabolites regulate interleukin-1 production. The physiologic, metabolic and immunologic action of interleukin-1. Ann Arbor, MI, June 1985, J. Leuk. Biol. 37, 721, 1985.

27. O'Flaherty, J.T., Thomas, M.J., Lees, C.J., and McCall, C.E. Neutrophil aggregating activity of monohydroxyeicosatetraenoic acids. Am. J. Pathol. 104, 55, 1981.

28. Van de Stadt, K.D. Prostaglandins and leukotrienes in inflammation and allergy. Neth. J. Med. 25, 22, 1982.

29. Stenson, W.F., and Parker, C.W. Monohydroxyeicosatetraenoic acids (HETEs) induce degranulation of human neutrophils. J. Immunol. 24, 2100, 1980.

30. Kunkel, S.L., Chensue, S.W., Mouton, C., and Higashi, G.I. Role of lipoxygenase products in murine pulmonary granuloma formation. J. Clin. Invest. 74, 514, 1984.

31. Fukuhara, M., and Tsurufuji, S. The effect of locally injected anti-inflammatory drugs on carrageenin granulomas in rats. Biochem. Pharamacol. 18, 475, 1969.

32. Chensue, S.W., Quinlan, L., Higashi, G.I., and Kunkel, S.L. Role of oxygen reactive species in Schistosoma mansoni egg-induced granulomatous inflammation. Biochem. Biophys. Res. Commun. 122, 184, 1984.

33. Egan, R.W., Parton, J., and Kuehl, F.A., Jr. Mechanism of self-deactivation of prostaglandin synthetase. J. Biol. Chem. 251, 7329, 1976.

34. Humes, J.L., Sadowski, S., Galavage, M., Goldenberg, M., Subers, E., Bonney, R., and Kuehl, F.A., Jr. Evidence for two sources of arachidonic acid for oxidative metabolism by mouse peritoneal macrophages. J. Biol. Chem. 257, 1591, 1982.

35. Hsueh, W., Desai, U., Gonsalez-Crussi, F., Lamb, R., and Chu, A. Two phospholipase pools for prostaglandin synthesis in macrophages. Nature 290, 710, 1981. 